\title{
CARACTERÍSTICAS, DIFICULTADES Y NECESIDADES FORMATIVAS DE LOS PROFESORES TUTORES DE EDUCACIÓN INFANTIL Y PRIMARIA EN LOS CENTROS ESCOLARES DE LA PROVINCIA DE CASTELLÓN
}

\author{
CHARACTERISTICS, DIFFICULTIES AND TRAINING NEEDS OF \\ TUTOR-TEACHERS IN INFANT AND PRIMARY EDUCATION \\ SCHOOLS IN THE PROVINCE OF CASTELLÓN
}

\author{
Francisco-Juan García Bracete*, Rosa García Castellas** \\ y Fernando Doménech Betoret*** \\ Universidad Jaume I de Castellón
}

\begin{abstract}
RESUMEN
Hemos utilizado una muestra representativa de 100 profesores de 38 centros escolares públicos de infantil y primaria de la provincia de Castellón. Para categorizar las respuestas hemos adaptado el procedimiento basado en la teoría de la "fundamentación". Aunque la gran mayoría de profesores se consideran suficientemente preparados (88\%), más de la mitad afirman que no han recibido ninguna formación (58\%). La tutoría exige del maestro un conjunto de buenas características, especialmente tiene que ser un experto en relaciones interpersonales y tener una dedicación absoluta. Las relaciones con los padres y la falta de tiempo son las principales dificultades. Los tutores dicen que necesitarían mas formación práctica y conocimientos de psicología y pedagogía. La casi totalidad de categorías se han visto afectadas por algún factor del centro o del profesor, en particular la categoría que recoge el componente comunicador y negociador del tutor y las que hacen referencia a no tener dificultades.
\end{abstract}

\footnotetext{
* Francisco-Juan García Bracete, es Profesor Titular de Intervención en Ambientes Educativos de la U. Jaume I. Líneas de investigación: el estudio de los alumnos rechazados por sus compañeros, la participación de los padres en las escuelas y las relaciones familia-escuela y la formación del profesorado universitario.

** Rosa García Castellar, Es Profesora Asociada de la Universidad. Su campo de interés son las dificultades de aprendizaje en el área de las matemáticas y el estudio de los alumnos con trastorno de hiperactividad con déficit de atención e impulsividad.

*** Fernando Doménech Betoret, es Profesor Titular de Universidad en Psicología de la Instrucción. Sus trabajos se centran en el estudio de la situación educativa y en particular en el desarrollo de modelos de evaluación de los aprendizajes del alumno y de la actuación del profesor.
} 
Palabras clave: tutoría, profesor-tutor, características del tutor, formación del profesorado, necesidades formativas, educación infantil y primaria

\begin{abstract}
A representative sample of 100 teachers from 38 state-funded infant and primary schools in the province of Castellón was used in the study. We adapted the procedure based on grounded theory in order to classify replies. Although a very high percentage $(88 \%)$ of teachers consider themselves to be sufficiently well trained, more than half $(58 \%)$ stated that they had not received specific training. The role of tutor demands a wide range of good characteristics in the teacher, and in particular they have to be experts in interpersonal relationships and be absolutely dedicated to their jobs. The main difficulties are considered to be relationships with parents and lack of time. Tutors report a need for more practical training and psychopedagogical knowledge. Practically all categories have been affected by some school or teacher factor, in particular, the category covering the communicating and negotiating element of the role of tutor, and this referring to not having any difficulties.
\end{abstract}

Key words: tutorship, tutor-teacher, tutor characteristics, teachers training, formative gaps, infant and primary education

\title{
Introducción
}

En el año 1970 la Ley General de Educación introduce la tutoría en la enseñanza media de nuestro país. La LOGSE considera la tutoría como un factor de calidad de la enseñanza. El tutor es el encargado del "aprendizaje de ser persona", es el interlocutor entre el colegio y el alumno, los profesores y el alumno, el alumno y los padres, ...

Más de tres cuartas partes del profesorado afirma que un buen tutor influye positivamente en el rendimiento y en la madurez de los alumnos, y en torno al $60 \%$ que un mal tutor influye negativamente. Estos datos adquieren mayor relevancia cuando tomamos conciencia de que el $100 \%$ del profesorado de estos niveles ha sido o será tutor en alguna ocasión y que el $41 \%$ de los alumnos han tenido el mismo tutor durante toda la educación primaria (INCE, 2000).

En opinión de García Garrido (1999), la acción tutorial puede contribuir a dar una respuesta eficaz a dos de las tareas emergentes del profesor del siglo XXI, la tarea de adoptar decisiones sin colisiones y la tarea informativa. Ahora bien, como es sobradamente conocido los padres acuden poco al colegio, pero si asistieran lo que les pedimos quedarían defraudados y provocarían un atasco, porque el profesor (el tutor) no tiene ni el tiempo ni la formación ni la actitud necesarias.

En un clásico manual de tutoría, Román y Pastor (1984) clasifican las cualidades del tutor en tres grandes tipos: Cualidades humanas que dan respuesta a saber ser (empatía, madurez, sociabilidad, responsabilidad, aceptación), cualidades científicas que permiten saber (convivencia con el grupo, conocer y ayudar al alumno) y cualidades técnicas o saber hacer (trabajo en equipo, coordinar la orientación, ser eficaz). En opinión de Ortega (1994) dos cualidades pueden compendiar a todas las demás: empatía y comunicación.

La cualidad del tutor preferida por docentes, alumnos y padres es que el tutor se interese por sus alumnos (Ortega, 1994). En el mismo estudio también se destacan saber escuchar, ser justo, sincero, respetar las iniciativas de los alumnos. En 1981 este mismo autor 
preguntó qué cualidades debería tener el tutor. Los profesores destacaron principalmente la compresión (11\%), seguida de escuchar, interés por los alumnos, orientar a los alumnos y respeto (todas con el $8 \%$ ). No obstante resulta significativo que el $16 \%$ perteneciera a la categoría no sabe/no contesta. Pero, ¿qué cualidades dicen poseer los tutores mayoritariamente? Las respuestas de 48 profesores al cuestionario autodiagnóstico de Román y Pastor (1984) indican lo siguiente: me enriquezco con el trato con mis alumnos, soy hombre de equipo, estoy a disposición de mis alumnos, me es fácil establecer amistad con mis alumnos, me entusiasmo con mi trabajo, ansío hacer mejor mi trabajo. Entre las cualidades que dicen no poseer se encuentran tener sentido del humor, ser paciente y tener facilidad para tratar con los demás.

En la Comunidad Valenciana la formación de tutores no forma parte de los Planes de Estudio de Maestro; tan solo la asignatura optativa "acción tutorial y orientación psicológica familiar" de 4.5 créditos en la especialidad de Infantil de la Universidad de Alicante incorpora el término tutoría. Sólo el $22 \%$ del profesorado afirma que todo profesor está preparado para ser tutor (Ortega, 1994). En general, los profesores suelen señalar carencias en su formación, incluso hay quienes afirman, en el caso de secundaria, que sólo se debería acceder a la tutoría después de algunos años. El currículo del tutor está por elaborarse, pero, en opinión de Rus (1996), no se trata tanto de una preparación técnica, como de perfilar un estilo de actuación.

Cuando a los profesores se les pregunta porqué no han realizado (o lo han hecho de forma incompleta) algunas funciones adscritas al tutor suelen referirse a la falta de tiempo, preparación, experiencia, un lugar adecuado, medios, motivos, colaboración de las familias, pautas técnicas, o razones más específicas como falta de motivos para realizar las entrevistas con padres o riesgo de etiquetar o de favorecer el efecto halo al poner en común los conocimientos sobre el alumno en las reuniones de evaluación, etc., o no haber caído en la cuenta. La dedicación sobreañadida que supone la tutoría no es menor que la que exigen otros puestos de trabajos (INCE, 2000). En Educación Secundaria los tutores son conocidos como los "loqueros" y tutoría es sinónimo de sobrecarga de trabajo.

Pero la tutoría también es el cajón de sastre, mejor de desastre, de la educación formal (Ortega, 1994). A tan grandes retos, no le siguen el reconocimiento y valoración de la acción tutorial efectivamente desempeñada, la dedicación horaria es escasa (en la Comunidad Valenciana en el horario de los profesores de Educación Infantil y Primaria no se contempla la tutoría), no existen criterios para la selección de los tutores, no existen guías de elaboración de los Planes de Acción Tutorial, en adelante PAT, que suelen hacerse más en función de los listados teóricos de tareas administrativas que aparecen en las publicaciones oficiales o semioficiales que de su utilidad (García, Moreno y Torrego, 1996). En última instancia, todo queda bajo la responsabilidad y la buena voluntad del tutor.

De acuerdo con el estudio de Ortega (1994), profesores, alumnos y padres hacen las siguientes sugerencias de mejora: mejor preparación de los tutores (la más citada por profesores y alumnos), mayor organización, más dedicación, trabajo en equipo de profesores (la más citada por los padres), mayor interés del propietario (también participaban centros privados) y mayor contacto con los padres.

Dadas las importantes metas que se le encomiendan al tutor y de las innumerables dificultades que le acompañan parece interesante abordar el estudio del tutor. 


\section{Objetivos}

La finalidad de nuestro trabajo es aproximarse al estudio de la figura del tutor desde la perspectiva del profesor para poder contribuir a mejorar la situación actual. En este sentido pretendemos dar respuesta a las siguientes preguntas:

1. ¿Qué características ha de tener un buen tutor?

2. ¿Los profesores se consideran preparados para desarrollar la acción tutorial?

3. ¿Qué dificultades tienen los tutores?

4. ¿Los profesores han recibido formación para ejercer como tutor? En caso positivo, ¿qué tipo de formación han recibido?

5. ¿Qué necesidades formativas tienen los tutores?

6. ¿Existen diferencias en los objetivos anteriores en función de determinadas características del centro escolar (tamaño, ubicación rural o urbana, nivel sociocultural de las familias) o del profesor (género, edad, experiencia, antigüedad en el centro, formación, etapa educativa en la que imparte clase).

\section{Metodología}

\section{Muestra}

La muestra está formada por 100 profesores de educación infantil y primaria de la provincia de Castellón. Los 100 profesores, 76 profesoras y 23 profesores, con un rango de edad entre 24 y 60 años, ejercen su actividad en 38 centros escolares públicos de 19 localidades. El 66\% imparte clase en educación primaria (23, 18 y 25 en el ciclo inicial, medio y superior, respectivamente) y el $34 \%$ en educación infantil. Todos los entrevistados son diplomados en Magisterio; sólo 14 tienen estudios adicionales.

La muestra de profesores tiene una gran experiencia (82 tiene más de 10 años de experiencia; sólo 7 profesores no han cumplido todavía su primer quinquenio). La antigüedad en el centro también es elevada, el 54\% permanece en el centro actual más de cinco cursos, mientras que los nuevos sólo son 17.

El mayor porcentaje de profesores pertenece a poblaciones con más de 10.000 habitantes, 68 frente a 31 que lo hacen en zonas rurales. En cuanto al tamaño de los centros, 37 profesores trabajan en colegios de 9 ó menos unidades y 12 en colegios de 19 ó más unidades. El nivel sociocultural de las familias es fundamentalmente medio y medio-bajo (71\%).

\section{Instrumento}

Se ha utilizado un cuestionario formado por 125 preguntas (65 abiertas y 60 cerradas), distribuidas en 5 apartados generales. En el presente estudio sólo hemos empleado los datos de identificación, cuatro preguntas abiertas y dos cerradas del bloque "El Tutor":

- Pregunta 1: En su opinión, ¿qué características ha de tener el tutor?

- Pregunta 2: ¿Piensa que está preparado para desarrollar la acción tutorial? 
- Pregunta 3: Si corresponde, qué dificultades tiene usted como tutor/a?

- Pregunta 4: ¿Ha recibido algún tipo de formación para ejercer como tutor?

- Pregunta 5: En caso positivo, ¿qué formación ha recibido

- Pregunta 6: Si corresponde, ¿qué necesidades formativas tiene usted como tutor/a?

\section{Variables}

\section{a) Independientes}

- Ubicación del Centro Escolar. Se han establecido dos niveles: a) Rural: Localidades con menos de 10.000 habitantes; b) Urbano: localidades con más de 10.000 habitantes.

- Tamaño del Centro Escolar. Numero de Aulas. Se han seleccionado tres niveles: Pequeño (entre 7 y 9); Mediano (entre 10 y 18); Grande (más de 18).

- Nivel Sociocultural de las Familias. Se solicitó a los profesores que indicaran el nivel sociocultural de los familias de los alumnos que asisten al colegio: B) Bajo/bajo medio, M) Medio y A) medio-alto/Alto.

- Género del profesorado. Hombre, Mujer.

- Edad del profesorado. Número de años. Se han establecido 4 niveles de edad: 1) hasta 30 años, 2) entre 31 y 40 años, 3) entre 41 y 50 años, y 4) más de 50 años.

- Ciclo educativo en el que ejerce su labor docente. Se han realizado los siguientes agrupamientos: Etapa educativa (Infantil vs Primaria) (CICLO2) y Edad de los Alumnos (Pequeños: infantil $+1^{\circ}$ ciclo de primaria; Grandes: $2^{\circ}$ y $3^{\circ}$ ciclo de primaria) (CICLO3).

- Experiencia del profesorado. Años de experiencia como profesor. Tres niveles: Jóvenes (entre 1 y 4); Medios (entre 5 y 9), Experimentados (10 ó más).

- Antigüedad en un mismo centro. Años de permanencia en el centro escolar actual. Tres niveles: Noveles (primer año); Medios (entre 2 y 5); Antiguos (más de 5).

- Titulación académica. Recoge el grado de formación académica del profesorado. Se han realizado dos tipos de agrupamientos: a) Maestro vs profesor con Otros estudios (TITULACIÓN2) y b) Maestro vs. Licenciado (TITULACIÓN3).

\section{b) Dependientes}

- Características del tutor/a. Las 12 categorías y los agrupamientos resultantes del proceso de categorización de la pregunta 1.

- Preparación del profesor para actuar como tutor. Variable dicotómica (SI-NO) que mide si el profesorado se autopercibe formado para actuar como tutor.

- Tipo de dificultades del tutor/a. Las 11 categorías resultantes del proceso de categorización de la pregunta 3 .

- Formación recibida para ejerce como tutor. Variable dicotómica (SI-NO) que informa si se ha recibido formación en el ámbito de la acción tutorial. 
- Tipo de formación recibida. Indica los procedimientos de formación a los que se ha recurrido.

- Tipo de necesidades del tutor/a. Las 7 categorías resultantes del proceso de categorización de la pregunta 6 .

\section{Procedimiento}

Se contó con la colaboración de 15 entrevistadores. A cada entrevistador se le asignaron 3 centros escolares. La dirección del centro, una vez informada, seleccionaba tres o cuatro docentes del centro.

El estudio pretende ser representativo de la provincia de Castellón. Para ello se procedió a combinar una doble estrategia: extensiva, utilizando el máximo número de escuelas posible; intensiva, empleando un cuestionario que integre desde los aspectos generales de la acción tutorial a los más específicos. Ambas estrategias se compensaban entre sí.

\section{Análisis realizados}

1. Cálculo del porcentaje de respuestas para las preguntas 2,4 y 5 .

2. Proceso de categorización de las respuestas a las preguntas 1,3 y 6 .

3. Acuerdo interjueces en la clasificación de los enunciados en las categorías resultantes del proceso de categorización en cada una de las cuatro preguntas, mediante el cálculo del coeficiente Kappa.

4. Cálculo de porcentajes de las respuestas de cada categoría en función del profesorado que las aporta.

5. Calculo de la diferencia de medias entre cada par de categorías en cada una de las preguntas, mediante el cálculo de la "prueba t para muestras relacionadas" (no se presentan tablas).

6. Análisis diferencial en función de las variables del centro y del profesorado para cada una de las categorías de las preguntas 1,3 y 6, mediante la realización de ANOVAS de una vía.

\section{Proceso de categorización}

Para poder analizar las respuestas hemos procedido en primer lugar a categorizar las respuestas que nos ha dado el profesorado. Para ello hemos adaptado el procedimiento basado en la teoría de la "fundamentación" expuesto por Carrero (1999):

1. Transcripción literal de todas las respuestas emitidas por todos los profesores.

2. Primera clasificación, que agrupa tipos de respuestas semejantes (evitando clasificaciones "a priori").

3. Se inicia un nuevo análisis de las categorías comparando constantemente los tipos de respuestas y los agrupamientos generados (método comparativo constan- 
te). Las comparaciones son de tres tipos: respuesta/respuesta, respuesta/categoría y categoría/categoría. Este proceso de reclasificación continua hasta que las nuevas respuestas no añaden ninguna información significativa a las categorías generadas.

4. Se formula una definición de cada categoría, lo que provoca nuevos cambios.

5. Por último, se facilita a dos jueces independientes las definiciones de las categorías y la transcripción literal de los enunciados realizados por los profesores para que cada juez los clasifique en función de las definiciones.

\section{Resultados y Discusión}

\section{¿Cuáles son las características del tutor?}

- Descripción de las categorías. Del proceso de categorización han resultado 12 categorías, que pueden agruparse en otras 6 más generales:

A) Dedicación. Ejercer como tutor. Dedicación, responsable e interés por los alumnos.

1: Dedicación. Predisposición, entusiasmo, ganas de trabajar, que le guste la escuela, dedicar horas, motivación, vocación.

2: Responsable/profesional. Maestro preocupado, responsable, profesional.

3: Interés por los alumnos. Interés por los alumnos, prestar atención a los alumnos, querer a los alumnos, comportarse de forma afectuosa y cariñosa con los alumnos.

B) Comunicador/Negociador. Persona con cualidades comunicativas, con buen carácter, integradora y respetuosa.

4: Habilidades comunicativas. Facilidad para comunicarse, para hacerse entender y saber escuchar. Sociable, fiable y buen interlocutor.

5: Integrador. Persona abierta, con capacidad de adaptación y de integración, respetuosa y justa.

6: Buen carácter. Paciente, tranquila, positiva, amable, humana.

C) Sistemático-Resolutivo. Cualidades que reflejan orden y actividad. Sistemático y organizado, creativo y activo.

7: Sistemático. Sistemático, observador, ordenado, organizado.

8: Activo. Activo, resolutivo, reflexivo, creativo, participativo, con criterio.

D) Buen Modelo. Buen modelo en tanto que, por una parte, es coherente y equilibrado $y$, por otra, anima y orienta a los alumnos en una dirección.

9: Orientador. Dinamiza, estimula, motiva, transmite valores a los alumnos.

10: Equilibrado. Emocionalmente equilibrado, coherente, dominio de sí mismo.

E) Formación

11: Formación. Preparación, formación, conocimiento. Referencias a conocimientos psicológicos, pedagógicos, de las características de los niños. 


\section{F) Ninguna}

12: Ninguna. Indica que no hace falta ninguna característica específica o en especial.

- Acuerdos interjueces. Como vemos en la tabla 1 se alcanza un buen nivel de acuerdo entre los tres jueces. Los valores del coeficiente Kappa son .774 (juez 1 y 2), .818 (juez 1 y 3) y .795 (juez 2 y 3), todos con $p \leq .000$. Los tres jueces coinciden en 154 enunciados, lo que supone un porcentaje de acuerdo del $75.83 \%$.

TABLA 1: Características del tutor. Acuerdo interjueces.

\begin{tabular}{|c|c|c|c|c|c|}
\hline Jueces & $\mathbf{N}^{\mathbf{0}}$ de Casos & $\begin{array}{c}\mathbf{N}^{\mathbf{0}} \text { Casos } \\
\text { Acuerdo }\end{array}$ & \% de Acuerdos & $\begin{array}{c}\text { Coeficiente } \\
\text { Kappa }\end{array}$ & Significación \\
\hline 1 y 2 & 203 & 163 & 80.30 & .774 & .000 \\
\hline 1 y 3 & 202 & 170 & 84.16 & .818 & .000 \\
\hline 2 y 3 & 202 & 166 & 82.18 & .795 & .000 \\
\hline 1,2 y 3 & 203 & 154 & 75.86 & & \\
\hline
\end{tabular}

- Frecuencias y comparación entre categorías. Se han emitido 209 enunciados (201 válidos). La media, mediana y moda del $\mathrm{n}^{\mathrm{o}}$ de enunciados es de $2.01,2$ y 1 , respectivamente. Aunque la moda sea emitir enunciados pertenecientes a una sola categoría $(41.3 \%)$, la mitad de los profesores se refieren a dos o tres categorías distintas $(32.5 \%$ y $17.5 \%$, respectivamente). En la tabla 2 se observa las frecuencias de cada una de las 12 categorías y sus agrupamientos.

La categoría más frecuente es la que presenta al tutor como un profesor integrador y flexible $(E=55, P=33)$, que procura tener en cuenta todos los aspectos de la situación, ponerse en lugar de los demás, respetuoso y tolerante, abierto a las diferentes posibilidades, con flexibilidad en sus posiciones, que actúa con justicia. La capacidad de adaptación y de apertura son muy valoradas en el tutor. Estadísticamente esta categoría es significativamente superior a todas las demás categorías.

\section{"Persona abierta", \\ "Capacidad integradora", "Actitud empática".}

Las habilidades comunicativas $(E=27, P=24)$ ocupan el segundo lugar entre las preferencias del profesorado. Para poder actuar de forma equilibrada el profesor tiene que tener capacidades comunicativas, ser sociable, dialogante, saber conectar con los demás, que los demás le consideren un buen interlocutor, que le vean como una persona fiable y digna de confianza.

\section{"Talante tranquilo y dialogante", "Comunicativo", "La sociabilidad".}


TABLA 2: Frecuencias y porcentajes de las Categorías de "Características del Tutor".

\begin{tabular}{|l|c|c|c|}
\hline & \% Profesores & N Enunciados & \% Enunciados \\
\hline Dedicaci & 15 & 20 & 10,0 \\
\hline Responsb & 18 & 16 & 8,0 \\
\hline Interes & 14 & 16 & 8,0 \\
\hline Dedica2 & 39 & 52 & 25,9 \\
\hline Comunica & 24 & 27 & 13,4 \\
\hline Integrad & 33 & 55 & 27,4 \\
\hline Buencara & 11 & 13 & 6,5 \\
\hline Comunic2 & 49 & 95 & 47,3 \\
\hline Sistemat & 7 & 9 & 4,5 \\
\hline Activo & 11 & 13 & 6,5 \\
\hline Sist_Res & 17 & 22 & 11 \\
\hline Orienta & 9 & 12 & 6 \\
\hline Equilibr & 4 & 5 & 2,5 \\
\hline Buen_Mod & 12 & 17 & 8,5 \\
\hline Formacio & 12 & 13 & 6,5 \\
\hline Ninguna & 2 & 2 & 1 \\
\hline
\end{tabular}

Las dos categorías anteriores, junto con la categoría buen carácter, forman una macrocategoría (Comunica2, $E=95,47.26 \%, P=49$ ) que se diferencia significativamente de todas las demás. Esta macrocategoría presenta al tutor como un ser humano, experto en relaciones interpersonales, punto de encuentro de los intercambios comunicativos y referente para la solución de problemas.

La categoría buen carácter ocupa el séptimo lugar $(E=13, P=11)$. El tutor ha de ser optimista, positivo, para poder valorar a los demás, para ponerse en su lugar. El tutor ha de ser amable, tranquilo, paciente para dedicar el tiempo suficiente a relacionarse de forma tolerante, justa, para que se confíe en que ayudará a encontrar buenas soluciones.

\section{"Paciencia",$$
\text { "Positivo", }
$$$$
\text { "Persona humana". }
$$

A continuación nos encontramos con tres categorías que destacan ante todo la dedicación del profesor a su trabajo, que apelan a su responsabilidad y que exigen como condición que sienta interés y cariño por los alumnos (dedica2, $E=52,25.83 \% ; P=39$ ) Su probabilidad es significativamente más alta que la de las tres agrupaciones restantes. 
La acción tutorial exige del profesor que dedique tiempo a la tutoría $(E=20, P=15)$, que tengas ganas de trabajar, que le guste la escuela, que tenga entusiasmo y buena disposición. Se destaca la dedicación absoluta y vocacional del profesor.

\title{
"Estar pendiente del grupo", \\ "Buena disposición", \\ "Estar muy motivado".
}

A continuación, nos encontramos con una categoría en donde las palabras más mencionadas son responsabilidad y profesionalidad $(E=16, P=18)$. Se pide al tutor que asuma su responsabilidad con los alumnos y su entorno, que no escurra el bulto $\mathrm{y}$, ante todo, que se comporte con profesionalidad e integre los aspectos afectivos, e interpersonales. Se trata, en suma, de ser un buen maestro, un buen profesional.

$$
\begin{gathered}
\text { "Responsable”, } \\
\text { "Preocupación por el entorno familiar", } \\
\text { "Ser un buen profesional de la enseñanza". }
\end{gathered}
$$

La quinta categoría nos presenta a un profesor interesado por sus alumnos, preocupado por su bienestar, que crea un clima afectivo $(E=16, P=14)$. El profesor sitúa en primer lugar al alumno. El tutor ha de tener una buena relación con los alumnos, quererlos y tratarlos con afecto. Se compara al tutor con los padres.

\section{"Ha de interesarse por el bienestar de los alumnos", "Sensibilidad para valorar los caracteres de los alumnos", "Querer a sus alumnos".}

La macrocategoría "resolutivo/sistemático" aglutina las categorías "activo" y "sistemático". Sus 22 enunciados $(10.95 \%, P=17)$ aportan actividad y orden. Responder a las innumerables tareas del tutor le exigen ser activo y resolutivo, creativo y seguir un criterio respecto de la disciplina. Todo ello requiere que sea sistemático, actuar de forma ordenada y organizada. El tutor ante todo actúa permanentemente.

\author{
Sistemática (9). \\ "Ordenada", \\ "Organizada", \\ "Constancia". \\ Resolutiva/activo (13). \\ "Persona activa", \\ "Criterio claro respecto a normas y hábitos a realizar", \\ "Mente creativa".
}

La última agrupación de categorías, "Buen modelo" ( $E=17,8.46 \%, P=12)$, incluye las categorías "orientador" $(E=12, P=9)$ y la categoría "equilibrado" $(E=5, P=4)$. En ambas se transmite la idea de que el tutor ha de ser un buen modelo para el alumno: por una parte, ha de ser una persona emocionalmente equilibrada, con confianza y control de sí mis- 
mo; por otra parte, se espera que enseñe y de ejemplo de determinados valores, y que motive y oriente a los alumnos en su consecución.

\author{
"Persona motivadora", \\ "Orientador", \\ "Buen modelo a imitar". \\ "Emocionalmente equilibrada", \\ "Control de sí mismo", \\ "Confianza".
}

Aunque dos profesores creen que no se requiere ninguna característica específica o en especial, parecería bastante absurdo que así fuera ante el innumerable volumen de tareas que conforman la acción tutorial (García Bacete, García, Villanueva y Doménech, 2003). La categoría formación ( $E=13, P=12$ ) nos recuerda la necesidad de disponer de determinados conocimientos, en especial los propios de la psicología y de la pedagogía que nos permitirán conocer adecuadamente a los alumnos, y que estos conocimientos pueden aprenderse.

\title{
"Un maestro con formación psicológica y pedagógica", "Conocedor de las características del niño".
}

En síntesis, la tutoría exige que el maestro posea y muestre un amplio conjunto de características positivas. La tutoría es una tarea docente muy exigente. El tutor es un experto de las relaciones interpersonales, punto de encuentro de los intercambios comunicativos y referente para la solución de problemas. El tutor realiza una dedicación absoluta y vocacional. El tutor ha de ser resolutivo y sistemático, tiene que actuar permanentemente. El tutor debe ser un buen modelo, un buen profesional y poseer los conocimientos de los psicólogos.

\section{¿El profesorado se autopercibe preparado para actuar como tutor?}

La mayoría del profesorado considera que está preparado para realizar adecuadamente la acción tutorial (88\%). Tan sólo 3 profesores dicen que no lo están y 9 no contestan. Esta respuesta, pensamos, puede ir en la línea de no amenazar su autoeficacia, o de "hacemos lo que podemos dada nuestra formación y las facilidades de la administración". Esta imagen positiva no está influida por ninguno de los factores utilizados, por lo que podemos afirmar que se trata de una sensación generalizada entre el profesorado de infantil y primaria (Gráfica 1).

\section{¿Qué dificultades tienen los tutores?}

- Descripción de las categorías. Del proceso de categorización han resultado 7 categorías. El profesorado señala una amplia diversidad de situaciones que dificultan su labor. La categoría referida a las dificultades con los padres admite cinco subdivisiones.

1. Falta de experiencia o de formación (Dfalta_de). El profesorado indica los déficits en formación o la falta de experiencia suficiente. 


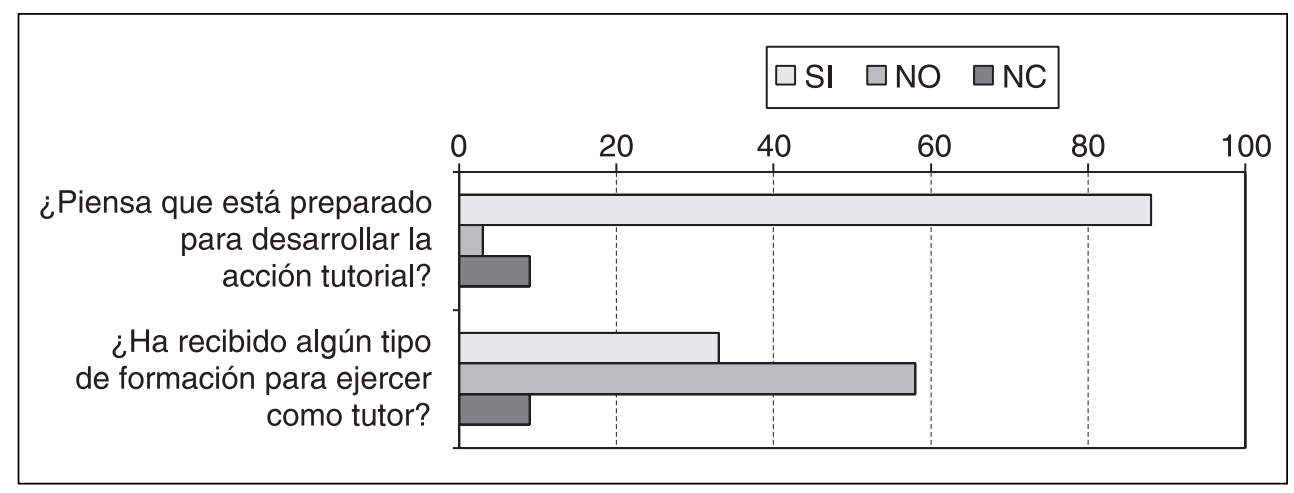

GRÁFICA 1.

Porcentaje de respuestas a los ítems 2 y 4.

2. Diversidad en el Aula (Ddiversid). Refleja el número excesivo de niños o la presencia de niveles diferentes dentro del aula.

3. Falta de tiempo (Dno_Tiemp). Expresa la falta de tiempo para realizar el número excesivo de tareas y la insuficiencia horaria.

4. Falta de trabajo conjunto (Dno_Equip). Se apunta la ausencia o niveles bajos de colaboración, bien con los compañeros bien con el equipo directivo.

5. Resolución de situaciones problemáticas en el aula (Dsitprob). Señala situaciones especiales para las que el profesor no se siente preparado o no dispone de los recursos suficientes (disciplina, integración, niños difíciles).

6. Trabajo con los padres (Dpadres). Incluye una serie de subdivisiones:

a) Falta de aprobación y de valoración (Dpnoapoyo). Recoge la falta de reconocimiento, de aprobación, la insatisfacción personal y económica.

b) Falta de interés de los padres (Dpnointer). Denuncia que las familias dedican poco tiempo a sus hijos, su falta de implicación y de interés.

c) Dificultades de comunicación con los padres (Dpnocomun). Expresa las dificultades de comunicación con las familias.

d) Falta de colaboración de las familias (Dpnocolab). Incluye la falta de respuesta de los padres, de colaboración o de continuidad.

e) Diferencia escuela/familia (Dpdies_Fa). Expresa interferencia o diferencia de valores entre la escuela y la familia. Caso particular de la división anterior.

7. Ninguna. Expresa la ausencia de dificultades o de alguna en especial.

- Acuerdo interjueces. Como vemos en la tabla 3 se alcanza un buen nivel de acuerdo entre los tres jueces. Los valores del coeficiente Kappa son .795 (juez 1 y 2), .778 (juez 1 y 3) y .809 (juez 2 y 3), todos con $p \leq .000$. Los tres jueces coinciden en 58 enunciados, lo que supone un porcentaje de acuerdo del $75.33 \%$. 
TABLA 3: Dificultades del Tutor. Acuerdo interjueces.

\begin{tabular}{|c|c|c|c|c|c|}
\hline Jueces & $\mathbf{N}^{\mathbf{0}}$ de Casos & $\begin{array}{c}\mathbf{N}^{\mathbf{C}} \text { Casos } \\
\text { Acuerdo }\end{array}$ & \% de Acuerdos & $\begin{array}{c}\text { Coeficiente } \\
\text { Kappa }\end{array}$ & Significación \\
\hline 1 y 2 & 77 & 63 & 81.82 & .795 & .000 \\
\hline 1 y 3 & 77 & 62 & 80.52 & .778 & .000 \\
\hline 2 y 3 & 77 & 64 & 83.12 & .809 & .000 \\
\hline 1,2 y 3 & 77 & 58 & 75.33 & & \\
\hline
\end{tabular}

- Frecuencias y comparación entre categorías. Se han emitido 80 enunciados (79 válidos). La media, mediana y moda del $\mathrm{n}^{\circ}$ de enunciados es de $.79,1$ y 0 , respectivamente. El $\mathrm{n}^{0}$ de profesores que han contestado es 57 , bastante bajo. La mayoría emiten un sólo enunciado $(42 \%)$ o se refieren a un sólo tipo de dificultad (49\%). En la tabla 4 se observa las frecuencias de cada una de las 11 categorías y sus agrupamientos correspondientes.

TABLA 4: Frecuencias y porcentajes de las Categorías de "Dificultades del Tutor".

\begin{tabular}{|l|c|c|c|c|}
\hline & \% Profesores & N Enunciados & \% total & \% clase \\
\hline Ninguna & 8 & 8 & 10,1 & \\
\hline Falta_de & 6 & 6 & 7,59 & \\
\hline Diversid & 5 & 5 & 6,33 & \\
\hline No_tiempo & 10 & 11 & 12,7 & \\
\hline No_equip & 3 & 3 & 3,80 & \\
\hline Sitprobl & 5 & 5 & 6,33 & \\
\hline Pnoapoyo & 5 & 5 & 7,59 & 14,3 \\
\hline Pnointer & 8 & 8 & 10,1 & 19 \\
\hline Pnocomun & 4 & 4 & 5,06 & 9,5 \\
\hline Pnocolab & 18 & 20 & 25,3 & 47,6 \\
\hline Pdies_fa & 4 & 4 & 5,08 & 9,5 \\
\hline Pdesencu & 20 & 24 & 30,4 & 57,1 \\
\hline Padres & 32 & 42 & 53,2 & 100 \\
\hline Novalida & 1 & 1 & 1,27 & \\
\hline
\end{tabular}

Los resultados apuntan a que los padres son el principal foco de las dificultades de los tutores:

a) sus 42 enunciados suponen el $53.16 \%$ del total,

b) el $56.14 \%$ de los docentes menciona una dificultad que tiene su origen en los padres, 
c) es significativamente superior a cualquier otra causa, con $p \leq .000$.

La categoría más numerosa es la que hemos denominado "desencuentro entre padres $y$ profesores" (30.38\%) formada principalmente por dos subconjuntos de enunciados: los que se refieren a la falta de colaboración por parte de los padres $(E=20, P=18)$ y los que evidencian interferencias y diferencias de valores entre la actuación de la escuela y de la familia $(E=4, P=4)$. Las dificultades para contactar y que los padres respondan de la forma esperada, o la falta de colaboración, de participación o de continuidad son las más numerosas. En muchas ocasiones los profesores especifican que se trata sólo de algunas familias o de familias con algunas características (desestructuradas, economía precaria, etc.) Se diferencia significativamente de las otras categorías, con niveles de significación entre $p \leq .016$ y $p \leq .000$.

\section{"Dificultad de contactar con algunas familias", \\ "Cuando los padres no colaboran",}

"Que a veces mi intervención no tiene continuidad en la familia del niño”.

"Incoherencia padre-tutor",

"La interferencia, en ocasiones de los padres".

Entre las 8 categorías restantes sólo se da alguna diferencia de probabilidad de forma aislada. Siguiendo con las otras categorías de padres, de mayor a menor frecuencia:

Falta de interés de los padres $(E=8, P=8)$, se refiere a la pobre implicación de las familias en la educación de los hijos, a su falta de interés y dedicación.

\section{"Falta de interés de los padres", "Indiferencia de los padres".}

Falta de valoración por parte de los padres $(E=6, P=4)$, expresa ataques a la autoestima del profesorado, mediante críticas injustificadas, prejuicios, no se valora la profesión o el trabajo realizado. Los tutores no se sienten apoyados por los padres.

\section{"Recibir la aprobación de todos en mi forma de trabajar", \\ "Ningún prestigio social de cara a los padres".}

Dificultades de comunicación con los padres $(E=6, P=5)$ expresa las dificultades para comunicarse con los padres de forma tranquila y eficaz, sobretodo en las situaciones en las que hay que dar informaciones negativas a los padres (el hijo presenta problemas, las expectativas de lo padres no se corresponden con la realidad) o en las que los padres desautorizan a los profesores, dando más crédito a lo que dice su hijo que a lo que el profesor les comenta.

\section{"Comunicarme con los padres", \\ "Actualmente creen más lo que sus hijos les dicen y suelen poner en tela de juicio la opinión del profesor".}

Entre los restantes focos de dificultades destaca la queja de falta de tiempo $(E=10$; $P=10)$. Los profesores dicen no disponer del tiempo suficiente para realizar todas las tareas o para desempeñarlas adecuadamente, que el tiempo de dedicación a la tutoría es insuficiente y no se contempla en la banda horaria ni del profesorado ni del alumnado. 


\section{"Falta de tiempo", \\ "Que el curriculum no contemple banda horaria", \\ "A veces, mucho papeleo".}

Otras fuentes de dificultades mencionadas por el profesorado son:

Falta de experiencia o de formación $(E=6, P=6)$. La cuarta fuente de dificultades es la experiencia insuficiente y la falta de oferta formativa institucional.

$$
\begin{gathered}
\text { "Me falta experiencia", } \\
\text { "Supongo que las propias de 'oficio",", } \\
\text { "Lo hago lo mejor que puedo, pero no sé si lo hago bien". }
\end{gathered}
$$

Incapacidad para hacer frente a determinadas situaciones problemáticas $(E=5, P=5)$, como las derivadas de la presencia de niños inmaduros, conflictivos, inmigrantes u otras problemáticas.

"Me resulta muy dificil integrar en el grupo sobretodo, algunos alumnos marroquíes",

"Como tratar a los niños con dificultades de comportamiento".

La problemática derivada de la diversidad de las aulas $(E=5, P=5)$. Las ratios elevadas o las aulas no graduadas son las principales fuentes de dificultades.

"Atender la diversidad en general",

"Diferentes niveles dentro del aula (4-5-6)",

"Exceso de niños para tutorizar bien".

La falta de trabajo conjunto $(E=3, P=3)$. Se apunta que en la práctica ni se trabaja en equipo ni los equipos directivos apoyan las iniciativas del profesorado.

"Falta de acción conjunta con otros compañeros de igual nivel",

"Poca colaboración del equipo directivo".

Finalmente, un $14.04 \%$ de los profesores que contestan $(E=8)$ afirman que el trabajo de la tutoría no les supone ninguna dificultad.

En síntesis, se puede afirmar que las relaciones con los padres supone la principal dificultad para los tutores. Más de la mitad de los enunciados emitidos presentan a los padres como un motivo de preocupación de los profesores. El sentido de dichos enunciados expresa un importante desencuentro con los padres o que los padres desatienden sus obligaciones. El resto de las dificultades apuntadas acentúan la falta de tiempo, la falta experiencias, la falta de habilidades y la falta de compromisos colectivos en el desempeño de la acción tutorial.

\section{¿Ha recibido algún tipo de formación para ejercer como tutor?}

La mayoría del profesorado dice que no han recibido ninguna formación (58\%). Si a estos 58 profesores incorporamos los 9 que no contestan, nos encontramos que dos de cada tres profesores no han recibido ninguna formación para desempeñar el rol de tutores (Tabla 5). 
TABLA 5: Tipos de Formación.

\begin{tabular}{|l|c|c|c|c|c|c|}
\hline & Ninguna & Estudios & Cursos & Autoformación & Compañero & Experiencia \\
\hline$\%$ Profesores & 23 & 14 & 16 & 5 & 8 & 12 \\
\hline
\end{tabular}

En cuanto a la formación recibida la mayoría de las respuestas apuntan hacia la formación recibida en los estudios de Magisterio o mediante la preparación de oposiciones o de lecturas. No obstante, la formula más utilizada es la asistencia a cursos de actualización. Aunque algunos se refieren a la experiencia acumulada como la mejor formación, bien como autorreflexión o como intercambio de opiniones, experiencias o recomendaciones entre compañeros.

La cuestión es si más de la mitad del profesorado no ha recibido formación como es que se consideran mayoritariamente preparados, ¿dónde y cómo han aprendido? Muchos señalan los estudios de magisterio o las oposiciones, cuando sabemos que los planes de magisterio no incluyen de forma explícita este curriculum. Tal vez estos resultados sólo expresen que la tutoría es una tarea menor en relación con la docente.

\section{¿Qué necesidades tienen los tutores?}

- Descripción de las categorías. Del proceso de categorización han resultado 7 categorías. El profesorado expresa un amplio número de necesidades diferentes en su desempeño profesional como tutor.

1. Todas/Siempre (Ntodas). Toda formación es necesaria y el proceso de mejora debe ser continuo.

2. Cursos (Ncursos). Se refiere a la necesidad de asistir a cursos de actualización que permitan adquirir destrezas y confianza.

3. Manuales (Nmanuales). Se reclama más libros o lecturas sobre la tutoría.

4. Intercambios (Nintercam). Se propone la necesidad de facilitar el intercambio entre compañeros en el centro u otros profesionales.

5. Experiencia (Nexperien). Destaca la necesidad de tener más experiencia.

6. Conocimientos (Nconocim). Se apunta la necesidad de adquirir conocimientos en determinados aspectos o áreas.

7. Ninguna. Ausencia de necesidades específicas para trabajar como tutor.

- Acuerdo interjueces. Como vemos en la tabla 6 se alcanza un buen nivel de acuerdo entre los tres jueces. Los valores del coeficiente Kappa son: .876 (juez 1 y 2), .835 (juez 1 y 3) y .959 (juez 2 y 3), todos con $p \leq .000$. Los tres jueces coinciden en 51 enunciados, lo que supone un porcentaje de acuerdo del $86.44 \%$.

- Frecuencias y comparación entre categorías. Los 42 profesores que contestan han emitido 58 enunciados. Se observa que el $73.8 \%$ emite un único enunciado y que el $83.3 \%$ sólo ofrece enunciados pertenecientes a una única categoría. El profesorado no se siente muy cómodo contestando este tipo de preguntas. En la tabla 7 se observa las frecuencias 
TABLA 6: Necesidades del Tutor. Acuerdo interjueces.

\begin{tabular}{|c|c|c|c|c|c|}
\hline Jueces & $\mathbf{N}^{\mathbf{0}}$ de Casos & $\begin{array}{c}\mathbf{N}^{\mathbf{0}} \text { Casos } \\
\text { Acuerdo }\end{array}$ & \% de Acuerdos & $\begin{array}{c}\text { Coeficiente } \\
\text { Kappa }\end{array}$ & Significación \\
\hline 1 y 2 & 59 & 53 & 89.83 & .876 & .000 \\
\hline 1 y 3 & 59 & 51 & 86.44 & .835 & .000 \\
\hline 2 y 3 & 59 & 57 & 96.61 & .959 & .000 \\
\hline 1,2 y 3 & 59 & 51 & 86.44 & & \\
\hline
\end{tabular}

de cada una de las 7 categorías, dos se refieren a la cantidad de formación y cinco al tipo de necesidad formativa. Empezando por la cantidad, nos encontramos con dos posiciones diferentes:

TABLA 7: Frecuencias y porcentajes de las Categorías de "Necesidades del Tutor".

\begin{tabular}{|l|c|c|c|}
\hline & \% Profesores & N Enunciados & \% Enunciados \\
\hline Ninguna & 7 & 7 & 12,07 \\
\hline Todas & 8 & 11 & 18,97 \\
\hline Cursos & 10 & 10 & 17,24 \\
\hline Manuales & 1 & 1 & 1,72 \\
\hline Intercam & 7 & 10 & 17,24 \\
\hline Experien & 4 & 4 & 6,90 \\
\hline Conocimi & 13 & 15 & 25,86 \\
\hline
\end{tabular}

a) El tutor debería participar en un proceso de formación continua y toda formación es necesaria y bien recibida $(E=11, P=8)$, incluso quien opina que se debería de realizar un periodo de "reprogramación" cada cierto tiempo.

\section{"Todas", \\ "Siempre es importante el intercambio de opiniones".}

b) La acción tutorial no requiere ninguna necesidad $(E=7, P=7)$, bien por el $n^{\circ}$ de años trabajados, bien porque no cree que exista una especialización para ser tutor.

\section{"Ninguna,}

"No creo que haya ninguna especialización para ser tutor".

Respecto de los tipos de formación, una categoría especifica los contenidos concretos en los que se es deficitario, mientras que las otras cuatro se refieren al canal utilizado.

Al profesorado le gustaría recibir preferentemente una formación aplicada sobre los contenidos propios de la psicología y la pedagogía y las nuevas tecnologías $(E=15$, 
$P=13)$. También se menciona el aprendizaje de formas efectivas de comunicación con las familias, la dinámica de grupos o la integración. Se diferencia estadísticamente de las categorías que especifican alguna modalidad formativa.

\section{"Psicología/Pedagogía actualizada y aplicada a las escuelas", \\ "Conocimiento de nuevas tecnologías", \\ "Como realizar una entrevista".}

La realización de cursos $(E=10, P=10)$ es sin lugar a dudas el procedimiento elegido mayoritariamente para dar respuesta a las necesidades. Se trata de formación breve, no necesariamente de tutoría.

\section{"Cursos de acción tutorial prácticos", "Cursos de perfeccionamiento".}

A continuación, nos encontramos con la propuesta de formación en centro y los intercambios $(E=10, P=7)$ : intercambio de opiniones, materiales y de experiencias entre compañeros del mismo y de distinto centro, formar parte de seminarios o realizar trabajos con los servicios psicopedagógicos o la universidad.

"A lo mejor se podría trabajar en seminarios",

"Los consejos de los compañeros son los que más ayudan",

"La realización de trabajo en equipo con la universidad".

Las otras dos fórmulas propuestas son prácticamente anecdóticas, por una parte cuatro profesores aluden a tener más experiencia, con una clara alusión a aquello de que el transcurrir de los años puede dar la sabiduría, y otro docente se refiere a la conveniencia de que existiera algún libro específico.

En síntesis, los datos nos muestran que la cuestión de la tutoría no está claramente definida, en tanto que nos encontramos que el profesorado expresa dos posiciones claramente diferenciadas: para unos la tutoría no requiere ninguna especialización, mientras que otros consideran que para actuar como tutor cualquier formación es poca. En particular, señalan los conocimientos en psicología y pedagogía como los más importantes y optan por los cursos de breve duración como el procedimiento preferido para dar respuesta a estas necesidades.

\section{Análisis diferenciales}

Se presentan los resultados de los ANOVAS de un factor en función de las siguientes variables del centro escolar (rural, tamaño, nivel sociocultural de las familias) y del profesorado (género, edad, experiencia, antigüedad en el centro, ciclo educativo y estudios). En las Tablas 8 a 15 las variables dependientes son las características del tutor. En las Tablas 16 a 24 las variables dependientes son la formación recibida, las dificultades y las necesidades detectadas. Por problemas de espacio sólo presentamos las diferencias con $p \leq .01$. 
TABLA 8: ANOVA Características del Tutor. Factor: RURAL.

\begin{tabular}{|c|c|c|c|c|c|c|c|c|}
\hline \multirow{2}{*}{ Variables } & \multicolumn{3}{|c|}{$\mathbf{U}$} & \multicolumn{3}{|c|}{$\mathbf{R}$} & \multirow{2}{*}{$\mathbf{F}$} & \multirow{2}{*}{$n$} \\
\hline & $\mathbf{N}$ & $\mathbf{X}$ & $\mathbf{S}$ & $\mathbf{N}$ & $\mathbf{X}$ & $\mathbf{S}$ & & \\
\hline Dedicaci & 68 & ,26 & 61 & 31 & ,06 &, 25 & 3,054 &, 084 \\
\hline Integrad & 68 & ,35 &, 71 & 31 & 1,0 & 1,1 & 12,81 & ,001 \\
\hline Dedica2 & 68 & ,68 & ,84 & 31 & , 19 & ,40 & 9,313 &, 003 \\
\hline Comunic2 & 68 & ,72 & 1,1 & 31 & 1,5 & 1,4 & 9,018 & ,003 \\
\hline
\end{tabular}

TABLA 9: ANOVA Características del Tutor. Factor: NUMERO DE UNIDADES.

\begin{tabular}{|c|c|c|c|c|c|c|c|c|c|c|c|}
\hline \multirow{2}{*}{ Variables } & \multicolumn{3}{|c|}{$\mathbf{P}$} & \multicolumn{3}{|c|}{ M } & \multicolumn{3}{|c|}{ G } & \multirow{2}{*}{$\mathbf{F}$} & \multirow{2}{*}{$p$} \\
\hline & $\mathbf{N}$ & $\mathbf{X}$ & $\mathbf{S}$ & $\mathbf{N}$ & $\mathbf{X}$ & $\mathbf{S}$ & $\mathbf{N}$ & $\mathbf{X}$ & $\mathbf{S}$ & & \\
\hline Equilibr & 37 &, 14 & 42 & 49 &, 00 &, 00 & 12 &, 00 & ,00 & 3,159 & 047 \\
\hline Interes & 37 & ,05 & 23 & 49 & 27 & 60 & 12 & ,08 & 29 & 2,399 & ,096 \\
\hline Buen_Mod & 37 & ,32 & 67 & 49 &, 10 & ,37 & 12 & ,00 & ,00 & 3,054 &, 052 \\
\hline
\end{tabular}

TABLA 10: ANOVA Características del Tutor. Factor: GENERO.

\begin{tabular}{|c|c|c|c|c|c|c|c|c|}
\hline \multirow{2}{*}{ Variables } & \multicolumn{3}{|c|}{ H } & \multicolumn{3}{|c|}{$\mathbf{M}$} & \multirow{2}{*}{$\mathbf{F}$} & \multirow{2}{*}{$p$} \\
\hline & $\mathbf{N}$ & $\mathbf{X}$ & $\mathbf{S}$ & $\mathbf{N}$ & $\mathbf{X}$ & $\mathbf{S}$ & & \\
\hline Dedicaci & 23 & ,39 & ,84 & 76 & , 13 & ,38 & 4,416 &, 038 \\
\hline Activo & 23 &, 00 &, 00 & 76 &, 17 & 47 & 2,987 & ,087 \\
\hline Formacio & 23 & ,26 &, 45 & 76 &, 08 & ,32 & 4,739 &, 032 \\
\hline Sist_Res & 23 & 00 & ,00 & 76 & 28 & ,60 & 4,808 & ,031 \\
\hline
\end{tabular}

TABLA 11: ANOVA Características del Tutor. Factor: EDAD.

\begin{tabular}{|c|c|c|c|c|c|c|c|c|c|c|c|c|c|c|}
\hline \multirow{2}{*}{ Variables } & \multicolumn{3}{|c|}{1} & \multicolumn{3}{|c|}{2} & \multicolumn{3}{|c|}{3} & \multicolumn{3}{|c|}{4} & \multirow{2}{*}{$\mathbf{F}$} & \multirow{2}{*}{$p$} \\
\hline & $\mathbf{N}$ & $\mathbf{X}$ & $S$ & $\mathbf{N}$ & $\mathbf{X}$ & $S$ & $\mathbf{N}$ & $\mathbf{X}$ & $\mathbf{S}$ & $\mathbf{N}$ & $\mathbf{X}$ & $\mathbf{S}$ & & \\
\hline Comunica & 7 & ,43 &, 53 & 26 & ,42 & ,58 & 39 & , 18 & ,39 & 20 & 20 & 31 & 2,743 &, 048 \\
\hline Comunic2 & 7 & 1,3 & ,76 & 26 & 1,4 & 1,5 & 39 & ,74 & 99 & 20 & ,40 & 82 & 3,762 & 014 \\
\hline
\end{tabular}

TABLA 12: ANOVA Características del Tutor. Factor: CICLO 3.

\begin{tabular}{|l|c|c|c|c|c|c|c|c|}
\hline \multirow{2}{*}{ Variables } & \multicolumn{3}{|c|}{ P } & \multicolumn{3}{|c|}{ G } & \multirow{2}{*}{ F } & \multirow{2}{*}{$\boldsymbol{p}$} \\
\cline { 2 - 8 } & $\mathbf{N}$ & $\mathbf{X}$ & $\mathbf{S}$ & $\mathbf{N}$ & $\mathbf{X}$ & $\mathbf{S}$ & & \\
\hline Activo & 53 &, 23 &, 54 & 43 &, 02 &, 15 & 5,664 &, 019 \\
\hline Sist_Res & 53 &, 32 &, 64 & 43 &, 09 &, 37 & 4,259 &, 042 \\
\hline
\end{tabular}


TABLA 13: ANOVA Características del Tutor. Factor: EXPERIENCIA.

\begin{tabular}{|c|c|c|c|c|c|c|c|c|c|c|c|}
\hline \multirow{2}{*}{ Variables } & \multicolumn{3}{|c|}{$\mathbf{J}$} & \multicolumn{3}{|c|}{ M } & \multicolumn{3}{|c|}{$\mathbf{E}$} & \multirow{2}{*}{$\mathbf{F}$} & \multirow[b]{2}{*}{$p$} \\
\hline & $\overline{\mathbf{N}}$ & $\mathbf{X}$ & $\mathbf{S}$ & $\mathbf{N}$ & $\bar{X}$ & $\mathbf{S}$ & $\mathbf{N}$ & $\mathbf{X}$ & $\mathbf{S}$ & & \\
\hline Buencara & 7 &, 00 & ,00 & 8 & ,38 & ,74 & 82 & ,09 & ,30 & 2,788 &, 067 \\
\hline Comunic2 & 7 & 1,7 & ,95 & 8 & 1,7 & 1,9 & 82 & ,74 & 1,1 & 4,331 &, 016 \\
\hline Integrad & 7 & 1,3 & ,95 & 8 & 1,0 & 1,3 & 82 & ,43 & ,79 & 4,620 &, 012 \\
\hline
\end{tabular}

TABLA 14: ANOVA Características del Tutor. Factor: ANTIGÜEDAD.

\begin{tabular}{|c|c|c|c|c|c|c|c|c|c|c|c|}
\hline \multirow{2}{*}{ Variables } & \multicolumn{3}{|c|}{$\mathbf{N}$} & \multicolumn{3}{|c|}{$\mathbf{M}$} & \multicolumn{3}{|c|}{$\mathbf{A}$} & \multirow{2}{*}{$\mathbf{F}$} & \multirow{2}{*}{$p$} \\
\hline & $\mathbf{N}$ & $\mathbf{X}$ & $\mathbf{S}$ & $\mathbf{N}$ & $\mathbf{X}$ & $\mathbf{S}$ & $\mathbf{N}$ & $\mathbf{X}$ & $\mathbf{S}$ & & \\
\hline Comunica & 17 & ,41 &, 51 & 23 &, 35 & ,49 & 54 &, 17 &, 42 & 2,506 & 087 \\
\hline Integrad & 17 &, 82 &, 88 & 23 &, 74 & 1,1 & 54 &, 35 &, 76 & 2,750 &, 069 \\
\hline Comunic2 & 17 & 1,4 & 1,1 & 23 & 1,3 & 1,4 & 54 & ,59 & ,96 & 4,421 &, 012 \\
\hline
\end{tabular}

TABLA 15: ANOVA Características del Tutor. Factor: TITULACION 3.

\begin{tabular}{|l|c|c|c|c|c|c|c|c|}
\hline \multirow{2}{*}{ Variables } & \multicolumn{3}{|c|}{ M } & \multicolumn{3}{|c|}{ L } & \multirow{2}{*}{ F } & \multirow{2}{*}{$\boldsymbol{p}$} \\
\cline { 2 - 7 } & $\mathbf{N}$ & $\mathbf{X}$ & $\mathbf{S}$ & $\mathbf{N}$ & $\mathbf{X}$ & $\mathbf{S}$ & & \\
\hline Orientad & 81 &, 09 &, 36 & 14 &, 29 &, 61 & 2,891 &, 092 \\
\hline
\end{tabular}

TABLA 16: ANOVA de Dificultades y Necesidades del Tutor. Factor: RURAL.

\begin{tabular}{|c|c|c|c|c|c|c|c|c|}
\hline \multirow{2}{*}{ Variables } & \multicolumn{3}{|c|}{$\mathbf{U}$} & \multicolumn{3}{|c|}{$\mathbf{R}$} & \multirow{2}{*}{$\mathbf{F}$} & \multirow{2}{*}{$p$} \\
\hline & $\mathbf{N}$ & $\mathbf{X}$ & $S$ & $\mathbf{N}$ & $\mathbf{X}$ & $\mathbf{S}$ & & \\
\hline Ddiversid & 68 & 01 &, 12 & 31 & ,13 & ,34 & 6,041 &, 016 \\
\hline Dpnoapoyo & 68 &, 01 &, 12 & 31 &, 16 &, 45 & 6,182 &, 015 \\
\hline
\end{tabular}

TABLA 17: ANOVA de Dificultades y Necesidades del Tutor. Factor: SOCIOCULTURAL.

\begin{tabular}{|c|c|c|c|c|c|c|c|c|c|c|c|}
\hline \multirow{2}{*}{ Variables } & \multicolumn{3}{|c|}{ B } & \multicolumn{3}{|c|}{ M } & \multicolumn{3}{|c|}{ A } & \multirow{2}{*}{$\mathbf{F}$} & \multirow{2}{*}{$p$} \\
\hline & $\mathbf{N}$ & $\mathbf{X}$ & $\mathbf{S}$ & $\mathbf{N}$ & $\mathbf{X}$ & $\mathbf{S}$ & $\mathbf{N}$ & $\mathbf{X}$ & $\mathbf{S}$ & & \\
\hline Ntodas & 37 & ,24 &, 49 & 34 &, 00 &, 00 & 11 &, 18 &, 40 & 4,073 &, 021 \\
\hline Nconocimi & 37 & ,03 &, 16 & 34 &, 21 & ,48 & 11 & ,45 & ,69 & 5,005 & ,009 \\
\hline
\end{tabular}


TABLA 18: ANOVA de Dificultades y Necesidades del Tutor. Factor:

NUMERO DE UNIDADES.

\begin{tabular}{|c|c|c|c|c|c|c|c|c|c|c|c|}
\hline \multirow{2}{*}{ Variables } & \multicolumn{3}{|c|}{$\mathbf{P}$} & \multicolumn{3}{|c|}{$\mathbf{M}$} & \multicolumn{3}{|c|}{ G } & \multirow{2}{*}{$\mathbf{F}$} & \multirow{2}{*}{$p$} \\
\hline & $\mathbf{N}$ & $\mathbf{X}$ & $\mathbf{S}$ & $\mathbf{N}$ & $\mathbf{X}$ & $\mathbf{S}$ & $\mathbf{N}$ & $\mathbf{X}$ & $\mathbf{S}$ & & \\
\hline Dpnoapoyo & 37 &, 16 & ,44 & 49 &, 00 &, 00 & 12 &, 00 &, 00 & 4,094 &, 020 \\
\hline Ncursos & 37 & , 19 & ,40 & 49 & ,06 & ,24 & 12 & 00 &, 00 & 2,727 & ,071 \\
\hline
\end{tabular}

TABLA 19: ANOVA de Dificultades y Necesidades del Tutor. Factor: GENERO.

\begin{tabular}{|l|c|c|c|c|c|c|c|c|}
\hline \multirow{2}{*}{ Variables } & \multicolumn{3}{|c|}{ H } & \multicolumn{3}{|c|}{ M } & \multirow{2}{*}{ F } & \multirow{2}{*}{$\boldsymbol{p}$} \\
\cline { 2 - 8 } & $\mathbf{N}$ & $\mathbf{X}$ & $\mathbf{S}$ & $\mathbf{N}$ & $\mathbf{X}$ & $\mathbf{S}$ & & \\
\hline Dninguna & 23 &, 17 &, 39 & 76 &, 05 &, 22 & 3,551 &, 062 \\
\hline Dpnocolab & 23 &, 35 &, 49 & 76 &, 16 &, 43 & 3,197 &, 077 \\
\hline
\end{tabular}

TABLA 20: ANOVA de Dificultades y Necesidades del Tutor. Factor: EDAD.

\begin{tabular}{|c|c|c|c|c|c|c|c|c|c|c|c|c|c|c|}
\hline \multirow{2}{*}{ Variables } & \multicolumn{3}{|c|}{1} & \multicolumn{3}{|c|}{2} & \multicolumn{3}{|c|}{3} & \multicolumn{3}{|c|}{4} & \multirow{2}{*}{$\mathbf{F}$} & \multirow{2}{*}{$p$} \\
\hline & $\mathbf{N}$ & $\mathbf{X}$ & $\mathbf{S}$ & $\mathbf{N}$ & $\mathbf{X}$ & $\mathbf{S}$ & $\mathbf{N}$ & $\mathbf{X}$ & $\mathbf{S}$ & $\mathbf{N}$ & $\mathbf{X}$ & $S$ & & \\
\hline Dninguna & 7 &, 00 & 00 & 26 &, 00 &, 00 & 39 &, 08 & 27 & 20 & 25 & 44 & 3,533 &, 018 \\
\hline
\end{tabular}

TABLA 21: ANOVA de Dificultades y Necesidades del Tutor. Factor: CICLO 3.

\begin{tabular}{|l|c|c|c|c|c|c|c|c|}
\hline \multirow{2}{*}{ Variables } & \multicolumn{3}{|c|}{ P } & \multicolumn{3}{c|}{ G } & \multirow{2}{*}{ F } & \multirow{2}{*}{$\boldsymbol{p}$} \\
\cline { 2 - 8 } & $\mathbf{N}$ & $\mathbf{X}$ & $\mathbf{S}$ & $\mathbf{N}$ & $\mathbf{X}$ & $\mathbf{S}$ & & \\
\hline Dno_Tiempo & 53 &, 04 &, 19 & 43 &, 16 &, 43 & 3,566 &, 062 \\
\hline Dpnointer & 53 &, 13 &, 34 & 43 &, 02 &, 15 & 3,747 &, 056 \\
\hline Nintercam & 53 &, 17 &, 55 & 43 &, 00 &, 00 & 4,159 &, 044 \\
\hline Nexperien & 53 &, 07 &, 27 & 43 &, 00 &, 00 & 3,437 &, 067 \\
\hline
\end{tabular}

TABLA 22: ANOVA de Dificultades y Necesidades del Tutor. Factor: EXPERIENCIA.

\begin{tabular}{|c|c|c|c|c|c|c|c|c|c|c|c|}
\hline \multirow{2}{*}{ Variables } & \multicolumn{3}{|c|}{$\mathbf{J}$} & \multicolumn{3}{|c|}{$\mathbf{M}$} & \multicolumn{3}{|c|}{$\mathbf{E}$} & \multirow{2}{*}{$\mathbf{F}$} & \multirow{2}{*}{$p$} \\
\hline & $\mathbf{N}$ & $\mathbf{X}$ & $\mathbf{S}$ & $\mathbf{N}$ & $\mathbf{X}$ & $\mathbf{S}$ & $\mathbf{N}$ & $\mathbf{X}$ & $\mathbf{S}$ & & \\
\hline Dfalta_De & 7 & ,43 & ,53 & 8 &, 00 &, 00 & 82 & ,04 &, 19 & 10,46 & ,000 \\
\hline
\end{tabular}


TABLA 23: ANOVA de Dificultades y Necesidades del Tutor. Factor: ANTIGÜEDAD.

\begin{tabular}{|c|c|c|c|c|c|c|c|c|c|c|c|}
\hline \multirow{2}{*}{ Variables } & \multicolumn{3}{|c|}{$\mathbf{N}$} & \multicolumn{3}{|c|}{ M } & \multicolumn{3}{|c|}{$\mathbf{A}$} & \multirow{2}{*}{$\mathbf{F}$} & \multirow{2}{*}{$p$} \\
\hline & $\mathbf{N}$ & $\mathbf{X}$ & $\mathbf{S}$ & $\mathbf{N}$ & $\bar{X}$ & $S$ & $\mathbf{N}$ & $\mathbf{X}$ & $\bar{S}$ & & \\
\hline Dninguna & 17 &, 00 & ,00 & 23 & 00 & 00 & 54 &, 15 & ,36 & 3,367 & 039 \\
\hline Dfalta_De & 17 & , 18 & 39 & 23 & ,09 & 29 & 54 & ,02 & , 14 & 2,921 & ,059 \\
\hline Nninguna & 17 & ,00 & ,00 & 23 & ,00 & 00 & 54 & , 13 & ,34 & 2,884 & 061 \\
\hline
\end{tabular}

TABLA 24: ANOVA de Dificultades y Necesidades del Tutor. Factor: TITULACIÓN 2.

\begin{tabular}{|c|c|c|c|c|c|c|c|c|}
\hline \multirow{2}{*}{ Variables } & \multicolumn{3}{|c|}{$\mathbf{M}$} & \multicolumn{3}{|c|}{$\mathbf{O}$} & \multirow{2}{*}{$\mathbf{F}$} & \multirow{2}{*}{$p$} \\
\hline & $\mathbf{N}$ & $\mathbf{X}$ & $\mathbf{S}$ & $\mathbf{N}$ & $\mathbf{X}$ & $\mathbf{S}$ & & \\
\hline Dnno_Tiemp & 81 & ,07 &, 31 & 17 & 24 & ,44 & 3,303 & ,072 \\
\hline Dpnocomun & 81 & ,02 & ,16 & 17 & , 12 & 33 & 3,137 & 080 \\
\hline
\end{tabular}

\section{A) Características del Tutor}

- Urbano-Rural. Los profesores rurales piden con más frecuencia que los urbanos que los tutores tengan habilidades comunicativas y estratégicas. En especial, las que caracterizan al tutor como integrador, abierto, tolerante. Por su parte, los profesores que trabajan en ambientes urbanos son los que más insisten, en el carácter vocacional de la tutoría.

- Tamaño del Centro Escolar. Los profesores de los colegios pequeños son los únicos que señalan la importancia del equilibrio emocional y del autocontrol del tutor. Que el tutor sea un buen modelo es una característica destacada en los colegios pequeños, llegando casi a desaparecer en los colegios grandes. Los profesores de los colegios medianos piensan que mostrarse afectivo y cariñoso con los alumnos es una característica importante. En los colegios pequeños y grandes prácticamente no se menciona esta característica.

- Género del tutor. Que el tutor muestre disponibilidad para pasar las horas necesarias en la escuela es más importante para los profesores varones que para las profesoras. También los varones destacan la importancia de la formación, pero, recordemos que el $94.1 \%$ del profesorado con otros estudios son mujeres. En sentido contrario, sólo las profesoras valoran un desempeño activo y sistemático de la tutoría, la organización de la tutoría y la creatividad, un sistema normativo bien pensado, que permita adoptar decisiones de forma rápida.

- Edad del tutor. Aunque la edad no aporta diferencias significativas, parece que los profesores más jóvenes (grupos 1 y 2) valoran más las habilidades comunicativas. Para los profesores jóvenes es importante saber conectar, que se les considere fiables. También conceden importancia a las destrezas que ayudan a resolver las situaciones problemáticas de forma empática y flexible.

- Ciclo Educativo. El profesorado que imparte clase en los primeros niveles educativos, sobretodo por el profesorado del ciclo inicial, prefiere una actividad tutorial dinámica, basada en el uso de normas trabajadas en clase. De la misma forma, el 
profesorado de estos niveles valora la actuación sistemática y ordenada del tutor. Ambas tendencias pueden estar asociados a la mayor proporción de mujeres en estos niveles.

- Experiencia docente. Los profesores noveles valoran las soluciones integradoras más que los otros grupos de experiencia docente. Se esfuerzan por tener en cuenta todos los puntos de vista. Por su parte, los profesores entre 5 y 9 años de experiencia son los que más importancia conceden a poder abordar las situaciones de forma tranquila y amable.

- Antigüedad en la Escuela actual. Lo dicho para los profesores jóvenes es válido para los profesores noveles en el centro. Para los profesores recién incorporados al centro es importante conectar con los demás, ser un interlocutor fiable y tolerante. La importancia de estas características diminuye con los años en el centro.

- Titulación. El profesorado de magisterio que es licenciado en psicología o pedagogía prioriza más que los otros docentes la habilidad para orientar, motivar y estimular a los alumnos y de transmitir determinados valores.

- En general, podemos afirmar que no existe una influencia muy marcada de los factores utilizados en la importancia concedida a unas características u otras. El mayor efecto se nota en la macrocategoría “comunica2". Los profesores varones, que trabajan en colegios rurales, en educación primaria, con menos de 40 años, con una experiencia inferior a 10 años y una antigüedad en el centro no superior a los 5 años son los que más valoran las habilidades comunicativas e integradoras en la solución de problemas. Los estilos activos son los preferidos por las profesoras y el profesorado de los niveles educativos más bajos. La predisposición es remarcada por los profesores varones y por los que trabajan en ciudades.

\section{B) Dificultades y Necesidades del Tutor}

- Urbano-Rural. Para los profesores rurales la falta de apoyo de los padres y la convivencia en la misma aula de alumnos de diversos cursos son sus principales dificultades.

- Sociocultural. En los ambientes socioculturales bajos perciben que necesitan de todo, que el número de sus necesidades son inabarcables. Por otra parte, de forma específica la necesidad de conocimientos se incrementa a medida que aumenta el nivel sociocultural de las familias.

- Tamaño del Centro Escolar. Los profesores de los colegios pequeños proclaman al mismo tiempo que no se sienten valorados por los padres y que es necesario relacionarse con los padres. Sin embargo, son los profesores de los colegios grandes los que incluyen entre sus funciones informar, orientar y favorecer la colaboración con los padres (García Bacete, Villanueva y Sorribes, 2003). También son los profesores de los colegios pequeños los que expresan más frecuentemente su necesidad de acceder a cursos de perfeccionamiento.

- Género del tutor. Una mayor proporción de profesores varones que de mujeres afirman que no tienen dificultades en la tutoría. Sin embargo, también son los profesores quienes más expresan dificultades para conseguir que los padres colaboren. 
- Edad del tutor. Con relación a la edad, todos los profesores que dicen no tener dificultades superan los 40 años, sobretodo los más mayores. Aquí parece valer aquello de "la edad tiene un grado"; entre los más jóvenes todos tienen al menos una dificultad.

- Ciclo Educativo. Los profesores de los ciclos medio y superior de primaria son los que más se quejan de la falta de tiempo. Por otra parte, el profesorado de infantil y del ciclo inicial es el que más insiste en la falta de interés de los padres por la educación de sus hijos, a pesar de contar con los niveles de colaboración escuela/familia más altos. El profesorado de infantil dicen necesitar más experiencia e incrementar los intercambios entre compañeros y con otros profesionales.

- Experiencia docente. Lógicamente, son los profesores noveles los que más insisten en que les falta experiencia (en una proporción de 12 noveles por cada experimentado). Parece que estuvieran diciendo que su principal problema radica en la permanente novedad en la que viven. Sin embargo, posiblemente ésta no sea la única explicación.

- Antigüedad en la Escuela actual. Los profesores nuevos en el centro también se quejan de la falta de experiencia. En sentido contrario, los más antiguos dicen no tener dificultades ni necesidades, se sienten integrados. La permanencia en la escuela parece ser un antídoto.

- Titulación. Los profesores que más frecuentemente encuentran dificultades cuando tratan de comunicarse con los padres son los que tienen más estudios, especialmente los profesores de pedagogía terapéutica. La falta de tiempo también está más extendida entre el profesorado con más estudios. Los nuevos conocimientos que les aporta los estudios les hace ser más sensibles a estas dificultades. Los estudios de psicología y de pedagogía han sido una salida para profesores inquietos.

En general, podemos afirmar que todos los factores empleados han mostrado tener un efecto en las variables medidas. A la inversa, la mayoría de variables han resultado influidas por un solo factor (14 de las 25 medidas). Proporcionalmente, el mayor número de diferencias se ha concentrado en el ámbito de las dificultades.

Las variables que han aglutinado el mayor número de diferencias han sido:

- Los profesores varones, más antiguos y de más edad informan más frecuentemente que no tienen ninguna dificultad.

- Los profesores noveles y nuevos en el centro proclaman su falta de experiencia.

- Los profesores que se sienten menos valorados por los padres trabajan en colegios pequeños ubicados en zonas rurales.

El plan de acción tutorial es una tarea docente de carácter prescriptivo. Al ejercicio de la tutoría se le concede una gran importancia y supone una tarea compleja, que conlleva el cumplimiento de numerosas funciones y la realización de un número de actividades todavía más extenso (García Bacete, García, Villanueva y Doménech, 2003). Por ello, no es de extrañar que numerosos estudios presenten la tutoría como un rol cuyo desempeño requiere de una adecuada preparación y que para actuar como tutor/a el profesorado tenga que activar múltiples cualidades y habilidades que suponen, entre otras cosas, que el profesor sea un experto en relaciones interpersonales y ejerza una dedicación absoluta (Ortega, 1994). 
Como hemos visto un alto porcentaje de profesores afirma que no ha recibido ninguna formación. Ni los planes de estudio de maestro ni la legislación educativa prestan atención a la formación de tutores ni a la organización de la acción tutorial. Coincidimos con Rus (1996) que sobretodo se trata de perfilar un estilo de actuación. Por ello pensamos que las necesidades y dificultades señaladas por los docentes en este estudio pueden ser una base adecuada para diseñar un plan de formación que permita al profesor adquirir y practicar las numerosas cualidades y habilidades que se exigen. De acuerdo con los resultados obtenidos, proponemos las siguientes dimensiones:

a) Organizativa: que posibilite disponer del tiempo necesario para planificar y ejercer la tutoría y para trabajar en equipo con los demás tutores y la jefatura de estudios. Tiempo y trabajo en equipo son dos ingredientes necesarios.

b) Gestión del aula: la respuesta a la diversidad y la resolución de conflictos en el aula serían los ejes fundamentales de esta dimensión.

c) Trabajo con familias: las dificultades de comunicación con los padres y la falta de colaboración de las familias son vistas por muchos profesores como una fuente de estrés y origen de gran parte de los problemas educativos. Poder contar con estrategias y contenidos que faciliten la incorporación de las familias a la vida cotidiana de los centros educativos es un objetivo claramente deseable (Forest y García Bacete, 2005).

Además, como los profesores afirman que gran parte de su formación procede de sus compañeros y de la experiencia, es decir, que compañeros y praxis son vistas como fuentes aceptables de formación, pensamos que la formación en centros y los modelos autoformativos y de reflexión conjunta sobre la propia práctica suponen una metodología adecuada para desarrollar dichos planes formativos. Con la ventaja adicional de permitir adecuarlos a los rasgos diferenciales que hemos visto, como pueden ser la etapa educativa, la formación previa, la experiencia docente o la ubicación del centro escolar en zonas rurales.

\section{Referencias bibliográficas}

Carrero, V. (1999). Análisis Cualitativo de Datos: Aplicación de la Teoría de la Fundamentación (grounded theory) en el ámbito de la innovación organizacional. Tesis Doctoral. Castellón: Universitat Jaume I.

Forest, C. y García Bacete, F. J. (2005). Comunicación cooperativa entre la familia y la escuela. Una guía para promover la colaboración entre profesores y padres. Valencia: Nau Llibres.

García Bacete, F. J., García, R., Villanueva, L. y, Doménech F. (2003). "Funciones y actividades que realizan los profesores-tutores en los centros escolares de infantil y primaria de la provincia de Castellón”. Revista Galego-Portuguesa de Psicoloxia e Educación, 7, Vol. 9, 113-126.

García Bacete, F. J., Villanueva, L. y Sorribes, S, (2003). "Funciones y actividades de tutoría en los centros escolares de infantil y primaria de la provincia de Castellón. Aspectos diferenciales en función del centro escolar y del profesorado". Revista Galego-Portuguesa de Psicoloxia e Educación, 7, Vol. 9, 97-111.

García Garrido, J. L. (1999). "El profesor del siglo XXI”. Bordón, 51 (4), 435-447.

García, R. J., Moreno, J. M. y Torrego, J. C. (1996). Orientación y tutoría en la educación secundaria. Madrid: Edelvives. 
INCE (2000). Sistema Estatal de Indicadores de la Educación. Madrid: Ministerio de Educación y Ciencia. Instituto Nacional de Calidad y Educación.

Ortega, M. A. (1994). La tutoría en secundaria obligatoria y bachillerato. Madrid: Editorial Popular y Fundación Hogar del Empleado.

Román, J. M. y Pastor, E. (1984, $3^{\mathrm{a}}$ Ed.) La tutoría. Pautas de acción e instrumentos útiles para el profesor-tutor. Barcelona: CEAC.

Rus, A. (1996). Tutoría, departamentos de orientación y equipos de apoyo. Granada: Servicio de publicaciones de la Universidad de Granada.

Fecha de recepción: 26-01-04

Fecha de revisión: 27-09-05

Fecha de aceptación: 19-10-05 